\title{
Limiares de reconhecimento de sentenças em indivíduos normo-ouvintes na presença de ruído incidente de diferentes ângulos
}

\author{
Sentences recognition thresholds in normal hearing individuals \\ in the presence of inciding noise from different angles
}

\author{
Marília Oliveira Henriques ${ }^{1}$, Maristela Julio Costa ${ }^{2}$
}

\begin{abstract}
RESUMO
Objetivo: Determinar e comparar os limiares de reconhecimento de sentenças no ruído, em campo livre, na presença de ruído incidente de diferentes ângulos e verificar qual a condição de escuta mais desfavorável, em indivíduos normo-ouvintes. Métodos: Aplicou-se o teste Listas de Sentenças em Português em 38 adultos jovens, avaliados em cabine acústica. As sentenças foram apresentadas a $0^{\circ}-0^{\circ}$ azimute e o ruído competitivo a $0^{\circ}-0^{\circ}, 0^{\circ}-90^{\circ}, 0^{\circ}-180^{\circ}$ e $0^{\circ}-270^{\circ}$ azimute, em intensidade fixa de $65 \mathrm{~dB}$ NPS (A). Resultados: As relações sinal-ruído nas quais foram obtidos os limiares de reconhecimento de sentenças no ruído para estes ângulos de incidência foram, respectivamente: $-7,56,-11,11,-9,75$ e -10,43 dB. Houve diferença entre os resultados nas condições: $0^{\circ}-0^{\circ}$ e $0^{\circ}-90^{\circ} ; 0^{\circ}-0^{\circ}$ e $0^{\circ}-180^{\circ} ;-0^{\circ}-0^{\circ}$ e $0^{\circ}-270^{\circ}$. Conclusão: Os seguintes limiares de reconhecimento de sentenças no ruído, em campo livre, foram obtidos nessas relações sinal/ruído: $0^{\circ}-0^{\circ}=-7,56 \mathrm{~dB} ;-0^{\circ}-90^{\circ}=-11,11 \mathrm{~dB} ;-0^{\circ}-180^{\circ}=-9,75 \mathrm{~dB} ; 0^{\circ}-270^{\circ}=$ $-10,43 \mathrm{~dB}$. Os melhores limiares no ruído foram obtidos com os ângulos de incidência de $0^{\circ}-90^{\circ}$ e $0^{\circ}-270^{\circ}$, seguidos pela condição de $0^{\circ}-180^{\circ} \mathrm{e}$, por último, $0^{\circ}-0^{\circ}$. A condição de escuta no ruído mais desfavorável foi aquela na qual o ruído encontra-se no mesmo ângulo de incidência da fala, na posição frontal do indivíduo avaliado.
\end{abstract}

Descritores: Testes de discriminação de fala; Ruído; Comunicação; Inteligibilidade da fala; Percepção da fala

\section{INTRODUÇÃO}

Para haver um processo de comunicação eficiente são necessários três elementos: emissor, mensagem e ouvinte. No entanto, a compreensão da mensagem, muitas vezes, é prejudicada pela presença de ruído competitivo. Esta situação dificulta a discriminação de estímulos de fala, não só para indivíduos normo-ouvintes, mas especialmente para os indivíduos com distúrbios da audição e usuários de próteses auditivas.

A fala é constituída por sons de frequências baixas e altas, que variam continuamente em intensidade, o que dificulta a predição do desempenho comunicativo do indivíduo apenas a partir dos limiares tonais registrados no audiograma ${ }^{(1)}$. Somando-se a esta consideração o fato de que grande parte

Trabalho realizado no Programa de Pós-Graduação em Distúrbios da Comunicação Humana, Universidade Federal de Santa Maria - UFSM - Santa Maria (RS), Brasil.

(1) Hospital Nossa Senhora da Conceição - HNSC - Porto Alegre (RS), Brasil.

(2) Curso de Fonoaudiologia, Universidade Federal de Santa Maria - UFSM - Santa Maria (RS), Brasil.

Endereço para correspondência: Marília Oliveira Henriques. R. Abram Goldsztein, 82/804 T3, Alto Petrópolis, Porto Alegre (RS), Brasil, CEP:

91450-155. E-mail: mariliahrq@yahoo.com.br

Recebido em: 14/2/2010; Aceito em: 13/7/2010 das situações comunicativas envolvendo a inteligibilidade da fala ocorre em ambientes ruidosos, verifica-se a necessidade de incluir, na rotina do fonoaudiólogo, testes que avaliem o paciente em condições mais próximas às que ocorrem no cotidiano. Para esta finalidade, testes de reconhecimento de sentenças no ruído são considerados ferramentas essenciais ${ }^{(2)}$.

Com esse propósito, nas últimas três décadas estes testes vêm sendo desenvolvidos e aprimorados, apresentando diferentes estratégias de aplicação ${ }^{(3-11)}$.

Uma destas estratégias consiste na apresentação dos estímulos de fala e ruído em campo livre, refletindo a forma como se estabelece a comunicação diária ${ }^{(12)}$. Para a avaliação em campo livre, a condição ideal é a apresentação dos estímulos de fala e de ruído a $0^{\circ}$ azimute, com localização da fonte sonora a um metro do indivíduo, por representar a situação mais comum de conversação ${ }^{(13)}$. No entanto, essa condição não permite que o indivíduo utilize sua habilidade de audição direcional binaural, que pode melhorar substancialmente o reconhecimento de fala no ruído, principalmente se houver diferença entre o ângulo de incidência da fonte sonora da fala e a do ruído competitivo ${ }^{(14)}$.

Ao considerar, então, que o ruído ambiental interfere de diferentes maneiras no reconhecimento da fala, dependendo 
da direção de sua incidência, os objetivos deste estudo foram: determinar e comparar os limiares de reconhecimento de sentenças no ruído, em campo livre, na presença de ruído incidente dos ângulos de $0^{\circ}-0^{\circ}, 0^{\circ}-90^{\circ}, 0^{\circ}-180^{\circ}$ e $0^{\circ}-270^{\circ}$ azimute e verificar qual foi a condição de escuta mais desfavorável, em indivíduos adultos jovens, normo-ouvintes.

\section{MÉTODOS}

Este estudo foi realizado no Ambulatório de Audiologia do Serviço de Atendimento Fonoaudiológico (SAF) da Universidade Federal de Santa Maria (UFSM), após registro no gabinete de projetos e aprovação no Comitê de Ética do Centro de Ciências da Saúde da Universidade Federal de Santa Maria (UFSM) sob número 115/04.

A amostra foi composta por 38 indivíduos normo-ouvintes, adultos jovens, sendo 18 do gênero masculino e 20 do gênero feminino. Foram avaliados somente os indivíduos que concordaram com a realização dos procedimentos necessários para a execução da pesquisa e que assinaram o Termo de Consentimento Livre e Esclarecido, após receberem maiores esclarecimentos sobre o objetivo e a metodologia do estudo proposto.

Como critérios de inclusão, adotou-se os resultados da Audiometria Tonal Liminar dentro dos padrões de normalidade nas frequências de $250 \mathrm{~Hz}$ a $8 \mathrm{kHz}^{(15)} \mathrm{e}$ a idade dos indivíduos, que deveria ser entre 18 e 35 anos.

Foram observados como critérios de exclusão a presença de alterações neurológicas, articulatórias e/ou de fluência verbal, presença de rolha de cerúmen ou de outras alterações no meato acústico externo capazes de alterar o desempenho no teste; dificuldade para memorizar as sentenças; também foram excluídos indivíduos normo-ouvintes que referiram dificuldade de compreensão da fala ou presença de zumbido.

As medidas desta pesquisa foram obtidas em cabine tratada acusticamente, utilizando-se um audiômetro digital de dois canais, marca Fonix ${ }^{\circledR}$, modelo FA-12, tipo I; fones auriculares tipo TDH- 39 P, marca Telephonics ${ }^{\circledR}$; um sistema de amplificação para audiometria em campo livre, com alimentação 110/220 Volts Ac, 50/60 Hz, potência de saída do amplificador de 80 Watts, potência das caixas de 100 Watts cada. As sentenças foram apresentadas utilizando-se um compact disc player Digital Toshiba ${ }^{\circledR}$ - 4149, acoplado ao audiômetro acima descrito; um medidor do nível de pressão sonora, digital, marca Radio Shack $®$.

Foi realizada a anamnese, por meio de um questionário constituído por questões fechadas, as quais forneceram informações referentes a dados pessoais, nível de escolaridade, profissão, hábitos de vida diária, história otológica e queixas auditivas dos sujeitos estudados. Estas informações foram levantadas para dar suporte à avaliadora durante o exame e para pesquisar possíveis critérios de exclusão, sem finalidade de análise posterior.

Realizou-se a inspeção visual do meato acústico externo, com a finalidade de excluir da amostra indivíduos que apresentassem alterações que pudessem interferir nos resultados das avaliações propostas.

Os pacientes foram submetidos à avaliação audiológica básica, constituída por: audiometria tonal liminar por via aérea nas frequências de $250 \mathrm{~Hz}$ a $8 \mathrm{kHz}$ e por via óssea nas frequências de $500 \mathrm{~Hz}$ a $4 \mathrm{kHz}$; pesquisa do limiar de reconhecimento de fala (LRF) e índice percentual de reconhecimento de fala (IPRF) apresentados em fones. Como as medidas posteriores seriam obtidas em campo livre, foi pesquisado também o LRF nesta condição, com apresentação das palavras a $0^{\circ}$ - $0^{\circ}$ azimute. Foram considerados como resultados dentro do padrão de normalidade audiometria tonal liminar com limiares até $25 \mathrm{~dB}^{(15)}$.

Os indivíduos selecionados foram avaliados para a obtenção dos limiares de reconhecimento de sentenças no ruído (LRSR), em campo livre. Para esta finalidade, foi aplicado o teste Listas de Sentenças em Português ${ }^{(3)}$, o qual é constituído por uma lista de 25 sentenças em Português Brasileiro ${ }^{(16)}$, sete listas com dez sentenças cada uma e um ruído com espectro de fala ${ }^{(17)}$. As sentenças e o ruído estão gravados em compact disc (CD), em canais independentes, e foram apresentados através de um CD player acoplado ao audiômetro.

Este teste é o pioneiro na utilização de sentenças para avaliação da comunicação de indivíduos, no Brasil. Seus critérios foram estabelecidos a partir de estudos ${ }^{(16,17)}$, com a descrição detalhada de todos os procedimentos usados e de trabalhos similares $^{(4-7)}$ realizados em outras línguas buscando critérios $^{(3)}$ adequados à realidade brasileira.

A aplicação do teste em campo livre foi realizada em ambiente acusticamente tratado, com o indivíduo posicionado a $1 \mathrm{~m}$ da fonte sonora, de frente para a mesma, inicialmente a $0^{\circ}-0^{\circ}$ azimute, ou seja, formando um ângulo de $0^{\circ}$ em relação aos planos horizontal e vertical.

Para responder ao teste, o indivíduo deveria repetir cada sentença da maneira que compreendeu, logo após a apresentação da mesma.

O sinal de fala foi mantido sempre na mesma posição, a $0^{\circ}$ - $0^{\circ}$ azimute. $O$ ruído competitivo sofreu alterações em sua posição de apresentação, formando ângulos de $0^{\circ}-0^{\circ}, 0^{\circ}-90^{\circ}$, $0^{\circ}-180^{\circ}$ e $0^{\circ}-270^{\circ}$ azimute em relação à posição da fonte sonora das sentenças.

O teste, em campo livre, foi realizado na seguinte sequência:

- apresentação das sentenças de 1 a 10 da lista $1 \mathrm{~A}$, a $0^{\circ}-0^{\circ}$ azimute, sem a presença de ruído competitivo, para familiarização do indivíduo com o teste;

- apresentação das sentenças de 11 a 20 da lista $1 \mathrm{~A}$, com a presença de ruído competitivo a $0^{\circ}-0^{\circ}$ azimute, para familiarização do indivíduo com o teste;

- apresentação da lista $1 \mathrm{~B}$ com presença de ruído a $0^{\circ}-0^{\circ}$ azimute;

- apresentação da lista $2 \mathrm{~B}$ com presença de ruído a $0^{\circ}-90^{\circ}$ azimute;

- apresentação da lista 3B com presença de ruído a $0^{\circ}$ - $180 .^{\circ}$ azimute;

- apresentação da lista 4B com presença de ruído a $0^{\circ}$ - $270^{\circ}$ azimute.

O motivo para a utilização de diferentes listas, uma para cada condição, foi a necessidade de eliminar a possibilidade de melhor desempenho devido à memorização das sentenças. $\mathrm{O}$ uso de listas diferentes não foi considerado como uma variável, 
pois, de acordo com um estudo anteriormente realizado ${ }^{(18)}$, todas são equivalentes entre si.

É importante salientar que a apresentação das sentenças de 1 a 10 da lista $1 \mathrm{~A}$, a $0^{\circ}-0^{\circ}$ azimute, sem a presença de ruído competitivo e a apresentação das sentenças de 11 a 20 da lista $1 \mathrm{~A}$, com a presença de ruído competitivo a $0^{\circ}$ - $0^{\circ}$ azimute foram utilizadas apenas com finalidade de treinamento, portanto, não foram consideradas na análise estatística.

Para a apresentação das sentenças, utilizou-se a estratégia sequencial ou adaptativa, ou ainda ascendente-descendente ${ }^{(19)}$, que permite determinar o limiar de reconhecimento de fala (LRF), que é o nível necessário para o indivíduo identificar, de forma correta, aproximadamente $50 \%$ dos estímulos de fala apresentados em uma determinada relação estímulo-resposta (S/R).

Neste procedimento, foi apresentado um estímulo em uma determinada relação $\mathrm{S} / \mathrm{R}$. Se o indivíduo fosse capaz de reconhecer corretamente o estímulo de fala apresentado, a intensidade do mesmo era diminuída em intervalos pré-estabelecidos, caso contrário, sua intensidade era aumentada. Este procedimento foi repetido até o final da lista. Uma resposta só foi considerada correta quando o indivíduo repetiu, sem erros ou omissões, a sentença apresentada.

A literatura ${ }^{(19)}$ sugere que sejam utilizados intervalos de 4 $\mathrm{dB}$ até a primeira mudança no tipo de resposta e, posteriormente, os intervalos de apresentação dos estímulos sejam de $2 \mathrm{~dB}$ entre si até o final da lista. Entretanto, devido às possibilidades técnicas do equipamento disponível para a realização desta pesquisa, foram utilizados intervalos de apresentação das sentenças de $5 \mathrm{~dB}$ e $2,5 \mathrm{~dB}$, respectivamente.

O nível da apresentação do ruído foi mantido fixo a $65 \mathrm{~dB}$ NPS $(\mathrm{A})^{(20)}$, sendo modificada a intensidade de apresentação das sentenças.

Os níveis de apresentação das sentenças foram anotados, para depois ser calculada a média, a partir dos valores onde houve mudança no tipo de resposta. Este valor foi subtraído do nível do ruído apresentado a 65 dB NPS (A), obtendo-se assim a relação $S / R$, na qual o indivíduo foi capaz de reconhecer em torno de 50\% dos estímulos apresentados.

Para a calibração dos estímulos em campo livre, estes foram medidos em nível de pressão sonora, utilizando a escala A do medidor, com respostas rápidas, que é a mais utilizada na mensuração de ruídos contínuos e para determinar valores extremos de ruídos intermitentes, além de ser a escala usada pela maioria dos pesquisadores nesta área ${ }^{(4,7,20,21)}$. O medidor foi posicionado em um ponto médio entre as duas orelhas, a uma distância de um metro do alto-falante.

A intensidade de apresentação das sentenças foi calibrada a partir de um tom puro registrado no canal do $\mathrm{CD}$ em que estão gravadas as sentenças. Esse tom puro, um som contínuo de referência, foi utilizado para que as mesmas condições de apresentação fossem mantidas. Antes do início das avaliações, a saída de cada canal do CD foi calibrada através do vumeter do audiômetro. Tanto o tom de $1 \mathrm{kHz}$, presente em um canal, quanto o ruído mascarante, presente no outro canal, foram colocados no nível zero. Estudos anteriormente realizados observaram que as sentenças foram gravadas no $\mathrm{CD}$ em uma intensidade média $7 \mathrm{~dB}$ abaixo da intensidade do tom puro ${ }^{(22,23)}$.
Essa diferença foi levada em consideração e corrigida no dial do equipamento no momento da aplicação dos testes.

A análise estatística dos resultados obtidos foi realizada da seguinte forma: inicialmente, os dados do grupo de homens e de mulheres foram analisados separadamente. Foi realizada a análise descritiva de ambos os grupos e, com a finalidade de verificar se os dados coletados seguiam uma distribuição normal, foi aplicado o teste de Shapiro Wilk, o qual constatou que as amostras, tanto do grupo de homens quanto do de mulheres, seguiam uma distribuição normal. A seguir, para verificar a existência de diferença de médias entre os grupos, em cada condição de teste, foi aplicado o teste " $t$ ". Este é um teste paramétrico, para verificar a existência de diferença de médias entre grupos independentes. Como não houve diferença estatistica entre os grupos $(p<0,05)$, os dados foram analisados conjuntamente, desprezando-se a variável gênero. Para analisar estes dados, foi realizada a análise estatística descritiva dos resultados obtidos nas quatro condições de teste $\left(0^{\circ}-0^{\circ}, 0^{\circ}-90^{\circ}, 0^{\circ}-180^{\circ}, 0^{\circ}-270^{\circ}\right.$, ) e, novamente, aplicado o teste de Shapiro Wilk para verificar a distribuição normal das amostras. Como, por este teste, os dados obtidos seguiam uma distribuição normal, as observações eram independentes e as variâncias dos grupos eram semelhantes, utilizou-se o teste paramétrico para diferença de médias por posição ANOVA. Neste teste, foram encontradas diferenças entre médias obtidas nas diferentes condições de avaliação. Para saber que grupos diferiam entre si, aplicou-se o teste de Scheffé. Para todos os testes aplicados, o nível de significância adotado foi $\alpha=0,05$.

\section{RESULTADOS}

Após verificar a normalidade de distribuição do grupo de homens e de mulheres $(p>0,05)$ e ao aplicar o teste paramétrico "t" para verificar existência de diferença entre as médias de ambos os grupos, não foi encontrada diferença quando considerada a variável gênero, os dados foram apresentados e analisados conjuntamente. A Tabela 1 apresenta a análise estatística descritiva das relações $\mathrm{S} / \mathrm{R}$ obtidas com o teste Listas de Sentenças em Português para todos os indivíduos examinados $(\mathrm{N}=38)$, nas condições de teste $0^{\circ}-0^{\circ}, 0^{\circ}-90^{\circ}$, $0^{\circ}-180^{\circ}$ e $0^{\circ}-270^{\circ}$.

Tabela 1. Análise estatística descritiva das relações $S / R(d B)$ obtidas nas diferentes condições de teste $(\mathrm{N}=38)$

\begin{tabular}{lcccc}
\hline & \multicolumn{4}{c}{ Condições de teste } \\
\cline { 2 - 5 } & $0^{\circ}-0^{\circ}$ & $0^{\circ}-90^{\circ}$ & $0^{\circ}-180^{\circ}$ & $0^{\circ}-270^{\circ}$ \\
\hline Média & $-7,563$ & $-11,116$ & $-9,751$ & $-10,430$ \\
Mediana & $-7,310$ & $-11,370$ & $-10,120$ & $-10,750$ \\
Mínimo & $-11,640$ & $-15,210$ & $-13,250$ & $-15,210$ \\
Máximo & $-4,770$ & $-6,640$ & $-5,880$ & $-7,000$ \\
Variância & 3,220 & 4,883 & 4,560 & 4,163 \\
DP & 1,795 & 2,210 & 2,135 & 2,040 \\
\hline
\end{tabular}

Legenda: $\mathrm{DP}=$ desvio-padrão

A Tabela 2 exibe os resultados do teste de Scheffé, no qual as condições de teste expressas como variáveis foram comparadas entre si para verificar quais diferiam estatisticamente. 
Sabendo-se que são considerados significativos os valores em que $\mathrm{p}<0,05$, assinalados com um asterisco na tabela, a variável $0^{\circ}-0^{\circ}$ diferiu das variáveis $0^{\circ}-90^{\circ}, 0^{\circ}-180^{\circ}$ e $0^{\circ}-270^{\circ}$. A variável $0^{\circ}-90^{\circ}$ diferiu das variáveis $0^{\circ}-0^{\circ}$ e $0^{\circ}-180^{\circ}$. A variável $0^{\circ}-180^{\circ}$ diferiu das variáveis $0^{\circ}-0^{\circ}$ e $0^{\circ}-90^{\circ}$. A variável $0^{\circ}-270^{\circ}$ diferiu somente da variável $0^{\circ}-0^{\circ}$.

Tabela 2. Comparação entre as relações S/R obtidas nas diferentes condições de teste

\begin{tabular}{lc}
\hline Variáveis & Valor de $p$ \\
\hline $0^{\circ}-0^{\circ}$ e $0^{\circ}-90^{\circ}$ & $0,00000^{*}$ \\
$0^{\circ}-0^{\circ}$ e $0^{\circ}-180^{\circ}$ & $0,00015^{\star}$ \\
$0^{\circ}-0^{\circ}$ e $0^{\circ}-270^{\circ}$ & $0,0000^{\star}$ \\
$0^{\circ}-90^{\circ}$ e $0^{\circ}-180^{\circ}$ & $0,04198^{*}$ \\
$0^{\circ}-90^{\circ}$ e $0^{\circ}-270^{\circ}$ & 0,54871 \\
$0^{\circ}-180^{\circ}$ e $0^{\circ}-270$ & 0,5576244 \\
\hline
\end{tabular}

*Valores significativos $(p \leq 0,05)$

\section{DISCUSSÃO}

Assim como no presente trabalho, outros estudos realizados em condições semelhantes ${ }^{(24,25)}$ verificaram diferença estatistica entre as relações sinal-ruído obtidas a $0^{\circ}-0^{\circ} \mathrm{em}$ relação às obtidas com ruído posicionado a $0^{\circ}-90^{\circ}$ e $0^{\circ}-270^{\circ}$ azimute, sendo estas últimas as condições mais favoráveis, nas quais houve melhora da inteligibilidade de fala no ruído. Isto ocorre porque, ao deslocar-se o ruído da posição frontal para a posição lateral do indivíduo, o estímulo sonoro apresentado lateralmente não é percebido igualmente pelas duas orelhas, ao contrário do que acontece quando a fonte sonora está localizada à sua frente. Essa diferença na percepção deve-se à presença de diferenças interaurais de tempo e de intensidade que ocorrem quando as fontes de fala e de ruído estão espacialmente separadas $^{(26)}$.

Um som que se origina do lado direito de um ouvinte, por exemplo, resulta na chegada deste som primeiro à orelha direita, pois está mais próxima da fonte sonora. Logo, após um breve intervalo, o som alcança a orelha esquerda, mais distante. Isto produz uma diferença interaural no tempo de recepção do som pelas duas orelhas. No entanto, a magnitude desta diferença de tempo interaural diminui com a mudança da fonte sonora da lateral $\left(0^{\circ}-90^{\circ}\right.$ ou $0^{\circ}-270^{\circ}$ azimute $)$ para a frente $\left(0^{\circ}-0^{\circ}\right.$ azimute $)$. Ou seja, quando o som se origina diretamente na frente do ouvinte, a distância para ambas as orelhas é a mesma e não há diferença de tempo interaural da chegada do som ${ }^{(27)}$.

Logo, a partir dessas informações, pode-se inferir que, quando o ruído é apresentado a $0^{\circ}-0^{\circ}$ azimute, juntamente com a fala, atinge ambas as orelhas simultaneamente. No entanto, quando é apresentado a $0^{\circ}-90^{\circ}$ ou $0^{\circ}-270^{\circ}$, atinge a orelha contrária com diferenças interaurais de tempo e de intensidade, fazendo com que esta orelha seja menos prejudicada pela presença do ruído competitivo, criando uma condição de escuta mais favorável. Desta forma, limiares mais elevados (piores) ocorrem quando as fontes de fala e de ruído se encontram na mesma posição e melhores limiares são encontrados quando a fonte da fala estiver localizada a $0^{\circ}$ diretamente em frente ao indivíduo e a fonte de ruído $90^{\circ}$ a sua direita ou esquerda ${ }^{(28)}$.

Outro ponto a ser discutido em relação aos dados encontrados é o fato dos valores obtidos nas condições de avaliação com ruído a $0^{\circ}-0^{\circ}$ e a $0^{\circ}-180^{\circ}$ diferirem estatisticamente, sendo que a melhor relação sinal-ruído foi obtida quando o ruído esteve localizado atrás do indivíduo. Apesar do ruído atingir as duas orelhas ao mesmo tempo, ao contrário do que ocorre quando incide lateralmente ao indivíduo, provavelmente sofre atenuação em função da posição anatômica do pavilhão auricular, que representará um obstáculo para a chegada do som emitido a $0^{\circ}-180^{\circ}$ até o conduto auditivo e a orelha média, o que não acontecerá com a fala emitida a $0^{\circ}-0^{\circ}$. Assim, essa condição de escuta será mais favorável do que a condição em que sentenças e ruído chegam ao mesmo tempo e com igual intensidade, como o que ocorre quando são ambos originados em fonte sonora localizada à frente do indivíduo.

A percepção espacial pode ser o fator de maior contribuição na experiência subjetiva da qualidade sonora. Afirma-se ainda, que a partir deste tipo de avaliação, temos possibilidade de explorar as habilidades da audição binaural do paciente e de selecionar próteses auditivas com características eletroacústicas que proporcionem melhor aceitação, auxiliando na reabilitação auditiva ${ }^{(29)}$. Sendo assim, pode-se dizer que a avaliação em campo livre, com separação espacial entre fala e ruído, tem contribuído para a seleção dos recursos tecnológicos mais apropriados para os casos em que a adaptação de aparelhos auditivos é necessária. Nessas situações, o resultado das avaliações irá influenciar diretamente na escolha do tipo de processamento do sinal e na seleção da tecnologia em microfones mais adequada para minimizar os efeitos do ruído competitivo no reconhecimento de fala do indivíduo.

Por estas razões, apesar destes testes exigirem tempo e habilidade do fonoaudiólogo, bem como disponibilidade e colaboração do paciente, é importante que sejam introduzidos na rotina clínica, sempre que possível. Através deles é possível avaliar com mais fidedignidade a habilidade de comunicação do indivíduo em condições que simulam situações do dia-a-dia. Porém, é importante considerar que, ao tratar dos distúrbios de audição, é necessário estabelecer valores de referência obtidos em indivíduos audiologicamente normais para poder, então, dimensionar as dificuldades encontradas pelo indivíduo com queixa de distúrbio de audição. Desta forma, a obtenção desses valores deve contribuir para novas pesquisas na área, servindo como parâmetro para estudos realizados nestas mesmas condições de avaliação, para diferentes grupos.

\section{CONCLUSÃO}

A partir da análise crítica dos dados, foi possível identificar os limiares de reconhecimento de sentenças no ruído, em campo livre, nas diferentes relações sinal/ruído. A comparação entre as condições avaliadas mostrou que os melhores limiares são obtidos com os ângulos de incidência de $0^{\circ}-90^{\circ}$ e $0^{\circ}-270^{\circ}$, seguidos pela condição de $0^{\circ}-180^{\circ}$ e, por último, $0^{\circ}-0^{\circ}$. Sendo assim, foi possível verificar que a condição de escuta no ruído mais desfavorável é aquela na qual o ruído encontra-se no mesmo ângulo de incidência da fala, na posição frontal do indivíduo avaliado. 


\begin{abstract}
Purpose: To determine and compare the sentence recognition thresholds in the noise, in sound field, in the presence of incident noise from different angles, and to verify the most unfavorable hearing condition, in normal-hearing individuals. Methods: The Portuguese Sentences Lists test was carried out in 38 young adults, evaluated in acoustic booth. The sentences were presented at $0^{\circ}-0^{\circ}$ azimuth, and the competitive noise at $0^{\circ}-0^{\circ}, 0^{\circ}-90^{\circ}, 0^{\circ}-180^{\circ}$, and $0^{\circ}-270^{\circ}$ azimuth, in a fixed loudness of $65 \mathrm{~dB}$ NPS (A). Results: The signal-to-noise ratios in which the sentence recognition thresholds were obtained for these incidence angles were, respectively: $-7.56,-11.11,-9.45$, and $-10.43 \mathrm{~dB}$. Differences were found between the results in the conditions: $0^{\circ}-0^{\circ}$ and $0^{\circ}-90^{\circ}$; $0^{\circ}-0^{\circ}$ and $0^{\circ}-180^{\circ} ;-0^{\circ}-0^{\circ}$ and $0^{\circ}-270^{\circ}$. Conclusion: The following sentence recognition thresholds in the noise, in sound field, were obtained for these signal-to-noise ratios: $0^{\circ}-0^{\circ}=-7.56 \mathrm{~dB} ;-0^{\circ}-90^{\circ}=-11.11 \mathrm{~dB} ;-0^{\circ}-180^{\circ}=-9.75 \mathrm{~dB} ; 0^{\circ}-270^{\circ}=-10.43$ $\mathrm{dB}$. The better thresholds were obtained with the incidence angles of $0^{\circ}-90^{\circ}$ and $0^{\circ}-270^{\circ}$, followed by the $0^{\circ}-180^{\circ}$ condition, and, finally, by the $0^{\circ}-0^{\circ}$ condition. The most unfavorable hearing condition was that in which the noise was in the same incidence angle of the speech, in front of the evaluated subject.
\end{abstract}

Keywords: Speech discrimination tests; Noise, Communication; Speech intelligibility; Speech perception

\title{
REFERÊNCIAS
}

1. Russo ICM, Pereira LD, Carvallo RMM, Anastásio ART. Encaminhamentos sobre a classificação do grau de perda auditiva em nossa realidade. Rev Soc Bras Fonoudiol. 2009;14(2):287-8.

2. Theunissen M, Swanepoel de W, Hanekom J. Sentence recognition in noise: variables in compilation and interpretation of tests. Int J Audiol. 2009;48(11):743-57.

3. Costa MJ. Listas de sentenças em português: apresentação \& estratégias de aplicação na audiologia. Santa Maria: Gráfica e Editora Pallotti; 1998.

4. Kalikow DN, Stevens KN, Elliot LL. Development of a test speech intelligibility in noise using sentence materials with controlled word predictability. J Acoust Soc Am. 1977;61(5):1337-51.

5. Plomp R, Mimpen AM. Speech-reception threshold for sentences as a function of age and noise level. J Acoust Soc Am. 1979;66 (5):1333-42.

6. Hagerman B. Sentences for testing speech intelligibility in noise. Scand Audiol. 1982;11(2):79-87.

7. Nilsson MJ, Soli SD, Sullivan JA. Development of the Hearing in Noise Test for the measurement of speech reception threshold in quiet and in noise. J Acoust Soc Am. 1994;95(2):1085-99.

8. van Wieringen A, Wouters J. LIST and LINT: sentences and numbers for quantifyng speech understanding in severly impaired listeners for Flanders and Netherlands. Int J Audiol. 2008;47(6):348-55.

9. Huarte A. The Castilian Spanisch in noise test. Int J Audiol. 2008; 47(6):369-70.

10. Luts H, Boon E, Wable J, Wounters J. FIST: a French sentence test for speech intelligibility in noise. Int J Audiol. 2008;47(6):373-4.

11. Wong LL. The Cantonese hearing in noise test. Int J Audiol. 2008;47(6):388-90.

12. Dubno JR, Ahlstrom JB, Horwitz AR. Binaural advantage for younger and older adults with normal hearing. J Speech Lang Hear Res. 2008;51(2):539-56.

13. Bronkhorst AW, Plomp R. A clinical test for the assessment of binaural speech perception in noise. Audiology. 1990;29(5):275-85

14. Iorio MCM. Utilização dos testes de reconhecimento de fala no processo de seleção e adaptação de próteses auditivas. In: Schochat E, organizadora. Processamento auditivo. São Paulo: Lovise; 1996. p. 12537.

15. Davis H, Silverman RS. Hearing and deafness. 3a ed. New York: Holt, Rinehart \& Winston; 1970.
16. Costa MJ, Iorio MCM, Mangabeira-Albernaz PL. Reconhecimento de fala: desenvolvimento de uma lista de sentenças em português. Acta AWHO. 1997;16(4):164-73.

17. Costa MJ, Iorio MCM, Albernaz PLM, Cabral Júnior EF, Magni AB. Desenvolvimento de um ruído com espectro de fala. Acta AWHO. 1998;17(2):84-9.

18. Santos SN, Daniel RC, Costa MJ. Estudo da equivalência entre as listas de sentenças em português. Rev CEFAC. 2009;11(4):673-80.

19. Levitt H, Rabiner LR. Use of a sequential strategy in intelligibility testing. J Acoust Soc Am. 1967;42(3):609-12.

20. Festen JM, Plomp R. Effects of fluctuating noise and interfering speech on the speech-reception threshold for impaired and normal hearing. J Acoust Soc Am. 1990;88(4):1725-36.

21. Soli SD, Nilsson M. Assessment of communication handicap with the HINT. Hear Instrum. 1994;45(2):14-6.

22. Cóser PL, Costa MJ, Cóser MJS, Fukuda Y. Reconhecimento de sentenças no silêncio e no ruído em indivíduos portadores de perda auditiva induzida pelo ruído. Rev Bras Otorrinolaringol. 2000;66(4):362-70

23. Soncini F, Costa MJ, Oliveira TMT, Lopes LFD. Correlação entre limiares de reconhecimento de sentenças no silêncio e limiares tonais. Rev Bras Otorrinolaringol. 2003;69(5):672-7.

24. Soli SD, Agnew J, Mayhugh C, Nilsson M, Gelnett D. Assessment of binaural directional hearing in hearing aid fitting. In: Meeting American Academy of Audiology, 1995; Dallas. Proceedings. Dallas: HEI; 1995; $1-9$.

25. Gelfand SA, Ross L, Miller S. Sentence reception in noise from one versus two sources: effects of aging and hearing loss. J Acoust Soc Am. 1988;83(1):248-56.

26. Dubno JR, Ahistrom JB, Horwitz AR. Spectral contributions to the benefit from spatial separation of speech and noise. J Speech Lang Hear Res. 2002;45(6):1297-310.

27. Humes LE. Considerações psicoacústicas em audiologia clínica. In: Katz J. Tratado de audiologia clínica. 4a ed. São Paulo: Manole; 1999. p. $56-72$.

28. Soli SD, Wong LL. Assesment of speech intelligibility in noise with the Hearing in Noise Test. Int J Audiol. 2008;47(6):356-61.

29. Byrne D, Noble W. Optimizing sound localization with hearing aids. Trends Amplif. 1998;3(2):51-73. 